\title{
Pocket formation and the flame surface density equation
}

\author{
WOLFGANG KOLLMANN \\ MAE Department \\ University of California \\ Davis, CA 95616 \\ and
}

JACQUELINE H. CHEN

Combustion Research Facility, Mail Stop 9051

Sandia National Laboratories

Livermore, California 94551-0969

\section{SAND-- $98-8472 C$}

Conf 980804 .

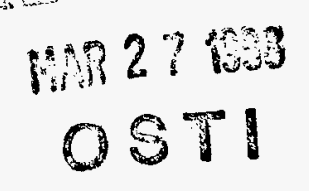

Corresponding author:

Wolfgang Kollmann

MAE Department

University of California

Davis, CA 95616, USA

Phone: (916) 752-1452

Fax: (916) 752-4158

email: wkollmann@ucdavis.edu

Word Count:

text: 3528 (estimate 392 words/page $\times 9.0$ pages)

figures: $1000(5 \times 200)$

total: 4528

Preferred Presentation:

Oral

Preferred colloquium topic area:

Turbulent flames (premixed)

Submitted to the 27th Symposium (International) on Combustion, Boulder, CO 


\section{DISCLAIMER}

This report was prepared as an account of work sponsored by an agency of the United States Government. Neither the United States Government nor any agency thereof, nor any of their employees, makes any warranty, express or implied, or assumes any legal liability or responsibility for the accuracy, completeness, or usefulness of any information, apparatus, product, or process disclosed, or represents that its use would not infringe privately owned rights. Reference herein to any specific commercial product, process, or service by trade name, trademark, manufacturer, or otherwise does not necessarily constitute or imply its endorsement, recommendation, or favoring by the United States Government or any agency thereof. The views and opinions of authors expressed herein do not necessarily state or reflect those of the United States Government or any agency thereof. 


\section{Abstract}

The occurrence and properties of singularities in the equation for the surface density function $\sigma \equiv|\nabla \Phi|$ are analyzed analytically and numerically using data from two-dimensional direct numerical simulation (DNS) of pocket formation in a premixed methane-air flame. The various stages and the relevant time scales associated with pocket formation were determined in a previous study. It was found that isolated pockets form if and only if a nondegenerate critical point of a saddle point type appears. The appearance of a singularity in the isoline representing the flame front may have implications to modeling of the terms in the surface density function (sdf) approach during such transient events as pocket formation. The sink and source terms in sdf are evaluated in the neighborhood of a critical point using DNS data during pocket formation, and an analytic representation of a scalar in the vicinity of the critical point which allows for the computation of all kinematic properties. The analytic and computational results show that the normal restoration and dissipation terms in the sdf become singular at the critical point when the pocket emerges. Furthermore, the analytic results show that the singularities exactly cancel, and therefore, the main conclusion is that it is unnecessary to model the singular behavior of these terms at critical points. However, closure of their sum is recommended. 


\section{Introduction}

Pocket formation is a highly transient and intermittent process in turbulent flames, that can lead to enormous values of flame stretch, rate-of-change of heat release and fuel consumption. In two dimensions, pocket formation involves propagating reacting isolines that mutually annihilate at a saddle point singularity of the scalar surface. Analysis of direct numerical simulation results and theory has demonstrated the roles of normal and tangential diffusion and reaction in determining the propagation of species isolines approaching and leaving the critical point during pocket formation in a premixed methane-air flame (Chen et al. [1]). In particular, it was determined that the following sequence of events characterize pocket formation: initially, prior to thermodiffusive interactions, normal diffusion is balanced by reaction in the primary reaction zone (channel region). However, normal diffusion and reaction become cumulative as a result of changes in species inflection points as the species diffusive layers merge. At the critical point the curvature, which was small in the channel region, becomes infinite as the approaching layers touch. At this point, both normal and tangential diffusion become unbounded, while the reaction rate vanishes. Retreating from the critical point, tangential diffusion at the cusps, which is linearly proportional to the local curvature, dominates normal diffusion. Subsequently, a reaction-diffusion balance is established as reaction rate at the cusps is restored on a time scale comparable with diffusion rates of radical species from the critical point to the cusps.

The focus of the present study is to determine the effect of pocket formation on the production and destruction mechanisms in the flame surface density equation. Models accounting for the contribution of intermittent, highly transient mutual annihilation events to dissipation and restoration of flame surface density do not exist. In flamelet models (Veynante et al. [2], Weller [3], Fichot et al. [4]), the flame surface density is one of the two main ingredients determining the local mean reaction rate. The combustion intensity, or the comsumption speed is the second ingredient. The results presented in this paper show that models for the singularity at critical points are not necessary. 


\section{SDF equation}

The flame surface density is defined as the differential surface area per unit volume (Candel and Poinsot [5], Cant et al. [6], Vervisch et al. [7]) by

$$
\Sigma \equiv|\nabla \Phi| \delta(\Phi-\varphi)
$$

which is governed by the balance equation (Bray [17])

$$
\frac{\partial \Sigma}{\partial t}+\frac{\partial}{\partial x_{i}}\left(v_{i} \Sigma\right)=\left(\frac{\partial v_{i}}{\partial x_{i}}-n_{i} n_{j} \frac{\partial v_{i}}{\partial x_{j}}\right) \Sigma-n_{i} \frac{\partial}{\partial x_{i}}\left\{\delta(\Phi-\varphi)\left[\frac{1}{\rho} \frac{\partial}{\partial x_{j}}\left(\rho \mathcal{D} \frac{\partial \Phi}{\partial x_{j}}\right)+\dot{\omega}\right]\right\} .
$$

The scalar $\Phi(\underline{x}, t)$ defining the flame surface is the solution of the transport equation

$$
\rho\left(\frac{\partial \Phi}{\partial t}+v_{i} \frac{\partial \Phi}{\partial x_{i}}\right)=\frac{\partial}{\partial x_{i}}\left(\rho \mathcal{D} \frac{\partial \Phi}{\partial x_{i}}\right)+\dot{\omega}
$$

with appropriate boundary and initial conditions. The unit normal vector of the surface is defined by

$$
n_{i} \equiv-\frac{1}{|\nabla \Phi|} \frac{\partial \Phi}{\partial x_{i}}
$$

The flame surface density defined above is a distribution and its derivatives in equation (2) must be regarded as generalized derivatives. It is preferable to work instead with the function $\sigma$ defined by

$$
\sigma \equiv|\nabla \Phi|
$$

Its transport equation follows at once from (3). It can be given in various forms, for instance

$$
\frac{\partial \sigma}{\partial t}+v_{i}^{*} \frac{\partial \sigma}{\partial x_{i}}=-n_{i} n_{j} \frac{\partial v_{i}^{*}}{\partial x_{j}} \sigma
$$

which is the most concise version, but not suitable for the investigation of the role of pocket formation since the velocity of the surface,

$$
v_{i}^{*} \equiv v_{i}+n_{i} S_{d}
$$

consisting of the fluid velocity, $v_{i}$, and the propagation velocity, $n_{i} S_{d}$ (Gibson, [8])

$$
S_{d} \equiv \frac{-1}{\rho|\nabla \Phi|}\left[\dot{\omega}+\frac{\partial}{\partial x_{i}}\left(\rho \mathcal{D} \frac{\partial \Phi}{\partial x_{i}}\right)\right]
$$


is not bounded at a critical point, and the unit normal vector is not defined. Hence, we work with the variant [9]

$$
\frac{\partial \sigma}{\partial t}+v_{i} \frac{\partial \sigma}{\partial x_{i}}=-n_{i} n_{j} \frac{\partial v_{i}}{\partial x_{j}} \sigma+n_{i} \frac{\partial}{\partial x_{i}}(\mathcal{D} \kappa \sigma)+n_{i} \frac{\partial}{\partial x_{i}}\left\{\frac{1}{\rho}\left[n_{j} \frac{\partial}{\partial x_{j}}(\rho \mathcal{D} \sigma)+\dot{\omega}\right]\right\}
$$

where the mean curvature of the flame surface is

$$
\kappa \equiv \frac{\partial n_{i}}{\partial x_{i}}
$$

The terms on the right hand side of (9) can be interpreted as (Peters [10])

production

$$
P \equiv-n_{i} n_{j} \frac{\partial v_{i}}{\partial x_{j}} \sigma
$$

dissipation

$$
D \equiv n_{i} \frac{\partial}{\partial x_{i}}(\mathcal{D} \kappa \sigma)
$$

and kinematic restoration

$$
R \equiv n_{i} \frac{\partial}{\partial x_{i}}\left[\frac{1}{\rho} n_{j} \frac{\partial}{\partial x_{j}}(\rho \mathcal{D} \sigma)\right]+n_{i} \frac{\partial}{\partial x_{i}}\left(\frac{\dot{\omega}}{\rho}\right) .
$$

It should be noted that neither production nor dissipation are definite; therefore, their sign may change.

\section{The effect of pocket formation on surface density}

Direct numerical simulation of reacting flows allows for the detailed analysis of the effect of pocket formation on the terms governing the surface density $\sigma$. Three distinct phases emerge from the results of the simulations. Isolated pockets can only be formed if a nondegenerate critical point of the type of a saddle point appears on the flame surface [1]. The associated time separates the three phases and is referred to as the critical time. First, we show analytically that dissipation and normal restoration become unbounded at the critical point, and then we present the results of the numerical simulations. The DNS is used to evaluate the terms in (9) before, during and after the isoline representing the flame surface approaches the critical point. The Lemma of Morse (see Milnor [11], lemma 2.2) implies 
that for a non-degenerate critical point of index unity (saddle point) the representation of the scalar

$$
\Phi(x, y)=\Phi_{0}-\frac{1}{2} x^{2}+\frac{1}{2} y^{2}
$$

holds (with appropriately scaled coordinates) in an open neighborhood of the critical point. The origin of the coordinate system is at the critical point and $\Phi_{0}$ is the value of $\Phi$ at the origin. This representation can be used to show that dissipation and kinematic restoration become unbounded at a non-degenerate critical saddle point, which is necessary for the formation of a pocket. Singularities in the right hand side of (9) for $\sigma$ must be expected for

pocket formation. The right hand side of the equation for $\sigma^{2} \equiv \nabla \dot{\Phi} \cdot \nabla \Phi$ is certainly bounded, $\frac{D \sigma^{2}}{D t}=R\left(\sigma^{2}\right)\left(D / D t\right.$ denotes the Stokes derivative) at critical points, but $\frac{D \sigma}{D t}=\frac{1}{2 \sigma} R\left(\sigma^{2}\right)$ has a singularity if $R\left(\sigma^{2}\right) \neq 0$ at a critical point. Hence, it is worth taking a closer look at the dynamics of $\sigma$ at critical points.

\section{Normal restoration at the critical point}

The kinematic restoration term, $R$, can be divided into normal and reactive contributions (13) and the normal restoration, $R_{N}$, (first term in (13)) can be further decomposed as

$$
R_{N}=n_{i} \frac{\partial n_{j}}{\partial x_{i}} \frac{1}{\rho} \frac{\partial}{\partial x_{j}}(\rho \mathcal{D} \sigma)+n_{i} n_{j} \frac{\partial}{\partial x_{i}}\left[\frac{1}{\rho} \frac{\partial}{\partial x_{j}}(\rho \mathcal{D} \sigma)\right] .
$$

It will be shown that both contributions in (15) are singular for $\sigma \rightarrow 0$. The reactive restoration (second term in (13)) remains bounded at a critical point. Hence, we need only analyze the two terms in the normal restoration. The scalar representation in (14) allows analytic computation of all kinematic terms in the neighborhood of the critical saddle point. From (5) and (14) we obtain $\sigma=\sqrt{x^{2}+y^{2}}$, and thus

$$
n_{i}=\frac{1}{\sigma}\left(\begin{array}{c}
x \\
-y
\end{array}\right)
$$

and

$$
\frac{\partial n_{i}}{\partial x_{j}}=\frac{1}{\sigma^{3}}\left(\begin{array}{cc}
y^{2} & -x y \\
x y & -x^{2}
\end{array}\right) .
$$

The divergence of the unit normal vector is the curvature, $\kappa \equiv \nabla \cdot \mathbf{n}$, and is given by

$$
\kappa=\frac{1}{\sigma} \frac{y^{2}-x^{2}}{y^{2}+x^{2}} .
$$


It follows from this result that the product $\kappa \sigma$ remains bounded as the critical point is approached. The first contribution to $R_{N}$ in (15), defined by

$$
R_{N 1}=n_{i} \frac{\partial n_{j}}{\partial x_{i}} \frac{1}{\rho} \frac{\partial}{\partial x_{j}}(\rho \mathcal{D} \sigma)
$$

is decomposed as

$$
R_{N 1}=\mathcal{D} n_{i} \frac{\partial n_{j}}{\partial x_{i}} \frac{\partial \sigma}{\partial x_{j}}+n_{i} \frac{\partial n_{j}}{\partial x_{i}} \frac{\sigma}{\rho} \frac{\partial}{\partial x_{j}}(\rho \mathcal{D}) .
$$

Using (16) and (17) we find that the second term is bounded at the critical point and we need only evaluate the first term. The resulting expression for $R_{N 1}$ is then

$$
R_{N 1}=\frac{4 \mathcal{D}}{\sigma} \frac{x^{2} y^{2}}{\left(y^{2}+x^{2}\right)^{2}}+n_{i} \frac{\partial n_{j}}{\partial x_{i}} \frac{\sigma}{\rho} \frac{\partial}{\partial x_{j}}(\rho \mathcal{D})
$$

It is easy to see that the first term becomes unbounded as the critical point (origin) is approached. For instance, choosing the path $x=y$ we get $\sigma=x \sqrt{2}$ and

$$
R_{N 1}=\frac{\mathcal{D}}{x \sqrt{2}}+\ldots \rightarrow \infty \text { as } x \rightarrow 0
$$

. The second contribution to the normal restoration

$$
R_{N 2}=n_{i} n_{j} \frac{\partial}{\partial x_{i}}\left[\frac{1}{\rho} \frac{\partial}{\partial x_{j}}(\rho \mathcal{D} \sigma)\right]
$$

is divided into singular and nonsingular contributions

$$
R_{N 2}=\mathcal{D} n_{i} n_{j} \frac{\partial^{2} \sigma}{\partial x_{i} \partial x_{j}}+n_{i} n_{j}\left\{\frac{\partial \sigma}{\partial x_{j}} \frac{\partial \mathcal{D}}{\partial x_{i}}+\frac{\partial}{\partial x_{i}}\left[\frac{\sigma}{\rho} \frac{\partial}{\partial x_{j}}(\rho \mathcal{D})\right]\right\} .
$$

The singular part of $R_{N 2}$ can be evaluated using (16) and the derivatives of $\sigma$ leading to

$$
\mathcal{D} n_{i} n_{j} \frac{\partial^{2} \sigma}{\partial x_{i} \partial x_{j}}=\frac{4 \mathcal{D}}{\sigma} \frac{x^{2} y^{2}}{\left(x^{2}+y^{2}\right)^{2}} .
$$

Near the critical point the normal restoration term behaves as

$$
R_{N}=\frac{8 \mathcal{D}}{\sigma} \frac{x^{2} y^{2}}{\left(x^{2}+y^{2}\right)^{2}}+\cdots
$$

where the dots indicate the nonsingular contributions. We conclude that normal restoration is singular at critical points and reactive restoration is bounded and, therefore, unimportant for the evolution of $\sigma$ during the formation of pockets. 


\section{Dissipation at the critical point}

Dissipation of $\sigma$ as defined above is not definite, but can be decomposed into a transport term and a negative definite true dissipation

$$
D=\frac{\partial}{\partial x_{i}}\left(\mathcal{D} \kappa \sigma n_{i}\right)-\mathcal{D} \sigma \kappa^{2} .
$$

Using the results obtained in the previous section for the restoration terms the transport term is obtained in the neighborhood of the critical point as

$$
\frac{\partial}{\partial x_{i}}\left(\mathcal{D} \kappa \sigma n_{i}\right)=\frac{\mathcal{D}}{\sigma} \frac{\left(x^{4}-10 x^{2} y^{2}+y^{4}\right)}{\left(x^{2}+y^{2}\right)^{2}} ;
$$

and the true dissipation term is

$$
\mathcal{D} \sigma \kappa^{2}=\frac{\mathcal{D}}{\sigma} \frac{\left(y^{2}-x^{2}\right)^{2}}{\left(x^{2}+y^{2}\right)^{2}} .
$$

The dissipation term behaves near the critical point according to

$$
D=-\frac{8 \mathcal{D}}{\sigma} \frac{x^{2} y^{2}}{\left(x^{2}+y^{2}\right)^{2}} .
$$

We conclude that the dissipation term, $D$, is also singular at critical points. Furthermore, the singular parts of the restoration and dissipation terms exactly cancel at the critical point.

\section{Numerical simulation results}

Direct numerical simulation is used to study the stages of pocket formation and their effect on the terms in the surface density equation for a premixed turbulent lean methane-air flame.

\section{Numerical Method}

The numerical scheme for the solution of the Navier-Stokes, species and energy equations for a compressible gas mixture is based on an explicit finite difference algorithm using a fourth-order low storage Runge-Kutta scheme for time advancement, and an eighth-order explicit spatial differencing scheme [12]. The chemical mechanism is based on a detailed $\mathrm{C}_{1}$ mechanism by Warnatz [13] with 17 species and 68 reversible reactions. The species 
mass diffusion is modeled with a Lewis number formulation and a prescription of the Lewis numbers for the different species [14]. The dynamic viscosity of the mixture is temperature dependent, while the thermodynamic properties (enthalpy, specific heat) are temperature and composition dependent. The Prandtl number is taken to be $\operatorname{Pr}=0.708$.

The computations are initialized with a one-dimensional steady laminar flame profile for a fuel-lean mixture (equivalence ratio of 0.7 ) of methane-air preheated to $800 \mathrm{~K}$. The profiles are obtained from a one-dimensional steady code PREMIX [15], and the solution is allowed to adjust to the simplified transport in a one-dimensional DNS.

The turbulence is prescribed by an initial two-dimensional turbulent kinetic energy spectrum function [16] which is superimposed on the laminar flame. The ratio of the turbulence intensity to the laminar flame speed, $u^{\prime} / s_{L}$, is taken to be ten and the ratio of the integral scale to the laminar flame thickness is $L_{11} / \delta_{F}=2.77$. Here, $\delta_{F}$ is a thermal thickness of the flame based on the maximum temperature gradient:

$$
\delta_{F} \equiv \frac{T_{b}-T_{u}}{(\mathrm{~d} T / \mathrm{d} x)_{\max }} .
$$

The subscripts $\mathrm{u}$ and $\mathrm{b}$ correspond to the unburned and burned states of the mixture. The turbulence Reynolds number based on $L_{11}$ and the unburned gas properties at $800 \mathrm{~K}$ is 181 . Unless otherwise noted, length scales are normalized by $\delta_{F}$, velocities by $s_{L}$, and time by flame time, defined as $\mathrm{t}_{F}=\delta_{F} / \mathrm{s}_{L}$. The computational domain size is $0.67 \mathrm{~cm}$, or $21.6 \delta_{F}$ in the directions parallel and perpendicular to the laminar flame. The domain is resolved into 750 uniform grid points in each direction.

\section{DNS results}

Two-dimensional unsteady DNS simulations of a premixed flame are performed with detailed chemistry to study the stages of pocket formation. After approximately 1 eddy turnover time, a pair of counter-rotating vortices embedded in the turbulent flow field, forms a thin channel of reactants as it passes through the flame. Subsequently an isolated unburnt pocket is created as the channel pinches-off, and a critical saddle point appears on the scalar surface chosen to represent the flame. The terms in the surface density equation are evaluated in the neighborhood of the critical points to establish the effect of the singularity on the dissipation 
and restoration terms. The emergence of the critical saddle point and the formation of the isolated pocket are illustrated using the $10 \%$ unburnt value of the methane mass fraction in Fig. 1. The terms in the surface density equation are then presented in Figs. 2 and 3 as the isoline approaches and leaves the saddle point.

\section{Prior to the critical time}

In Fig. 1 prior to the critical time, $\mathrm{t}=-0.02 t_{c} / t_{F}$, the methane isoline shows a narrow channel formed by the passage of a vortex pair. The corresponding sink and source terms in (9) are shown in Fig. $2 \mathrm{a}$ as a function of the arclength, in the neighborhood of where the channel pinches-off. Dissipation is negligible at this location, since the local curvature is small. As shown in this figure, reactive and normal restoration act in opposite directions, with normal restoration being much larger than reactive restoration. Production at this The main mechanism for destruction of $\sigma$ is due to normal restoration.

\section{At the critical time}

At the critical time the methane isolines from opposite sides of the channel touch at their point of tangency as shown in Fig. 1. At the critical point an isolated pocket of reactants emerges and two cusps are formed. As shown in Fig. 3b at this time, the terms in the surface density equation show a dramatic increase in both normal restoration ( $\cdots \cdots . .$. line) and dissipation (-- line). Furthermore, the increase in normal restoration is accompanied by a reversal in its sign. Analytically it was shown that the singularity in dissipation identically cancels the singularity in normal restoration; however, due to finite numerical discretization, it is not possible to show this numerically, although the width and magnitude of the two terms are approximately the same. Consistent with the analytic description, both production and reactive restoration are zero at the critical point.

\section{After the critical time}

The cusps that were formed during channel closing rapidly retreat from the critical point as shown in Fig. 1 at $\mathrm{t}=0.02 \mathrm{t}_{c} / \mathrm{t}_{F}$. As shown in Fig. $2 \mathrm{c}$ at an even later time, $\mathrm{t}=0.36 \mathrm{t}_{c} / \mathrm{t}_{F}$, dissipation becomes larger than normal restoration due to increasing values of $\sigma$ as the 
separated parts of the isosurface (isolines in 2-d) propagate away from the critical region. Normal restoration, on the other hand, counterbalances dissipation, although dissipation temporarily exceeds normal restoration as the cusps retreat away from the critical point. Reactive restoration and production are both negligible compared to diffusion. The rate at which the instantaneous surface density decreases then is determined by the ratio of dissipation to normal restoration. Eventually, an equilibrium is reached between these two processes as dissipation decreases and normal restoration once again becomes negative. At this point the cusps no longer retreat.

The analytic and numerical results indicate that the singular contributions to normal restoration and dissipation must cancel. This is evident in Figs. $2 b$ and $2 c$ and Fig. 3 (flame normal profile along $\mathrm{n}_{1}$ in Fig. 1), where the spokes clearly cancel. This result has an important consequence for the modeling of the normal restoration and dissipation terms in the surface density equation (9). It follows from the analytic investigation that normal restoration and dissipation terms are singular at critical points, but their sum is bounded since the singular contributions cancel out. Hence, it is not necessary to model the singular parts and only the nonsingular contributions require closure. This could be attempted by modeling the sum of normal restoration and dissipation, which is bounded at critical points.

\section{Conclusions}

The numerical and analytic investigation of pocket formation in a premixed methane-air flame shows that isolated pockets can form in the presence of strong vorticity. The formation of pockets is initiated by the emergence of narrow channels, which close and a critical saddle point appears. After the appearance of the critical point cusps form, which rapidly retreat and the pocket burns out subsequently. The normal restoration and dissipation terms in the equation for the surface density become singular at the critical point, but their sum remains bounded. This was demonstrated numerically and shown analytically. Hence, it is not necessary to model the singular behavior of these terms at critical points; rather, closure of their sum is recommended. 


\section{Acknowledgements}

This research was supported by the United States Department of Energy, Office of Basic Energy Sciences, Chemical Sciences Division.

\section{References}

[1] Chen, J. H., Echekki, T. and Kollmann, W. (1997), "The mechanism of two-dimensional pocket formation in lean premixed methane-air flames with implications to turbulent combustion", submitted to Combust. Flame, (1997).

[2] Veynante, D., Lacas, F., Candel, S., AIAA 89-0487 (1989).

[3] Weller, H. G., "The development of a new flame area combustion model using conditional averaging", Report TF/9307, Imperial College, London, 1993.

[4] Fichot, F., Delhaye, B., Veynante, D. and Candel, S. M., Twenty-fifth (Int.) Symposium on Combustion, The Combustion Institute, 1994, pp. 1273-1281.

[5] Candel, S. M. and Poinsot, T. J., Combust. Sci. E' Tech. 70:1-15 (1983).

[6] Cant, R. S., Pope, S. B. and Bray, K. N. C., Twenty-third (Int.) Symposium on Combustion, The Combustion Institute, 1990, pp. 809-815.

[7] Vervisch, L., Bideaux, E., Bray, K. N. C. and Kollmann, W., Physics of Fluids 7:2496$2503(1995)$.

[8] Gibson, C.H., Physics of Fluids 11:2305-2317 (1968).

[9] Van Kalmthout, E., Veynante, D., Candel, S. (1996), Twenty-sixthh (Int.) Symposium on Combustion, The Combustion Institute, 1996, pp. 35-42.

[10] Peters, N. Private communication, 1997.

[11] Milnor, J., Morse theory, Princeton University Press, 1963.

[12] Kennedy, C. A. and Carpenter, M. H., Appl. Num. Math., 14:3 (1996). 
[13] Warnatz, J., Maas, U., and Dibble, R., Combustion: Physical and Chemical Foundations, Modelling, Pollutant Formation, Springer-Verlag, Heidelberg, Germany, 1996.

[14] Smooke, M. D. and Giovangigli, V., in Reduced kinetic mechanisms and asymptotic approximation for methane-air flames (M.D. Smooke, Ed.), Lecture Notes in Physics 384, pp. 1-28, Springer-Verlag, New York, 1991.

[15] Kee, R. J., Grcar, J. F., Smooke, M. D. and Miller, J. A. Sandia National Laboratories Report SAND85-8240, 1985.

[16] Hinze, J. O. Turbulence, McGraw Hill, New York, 1975.

[17] Bray, K.N.C. (1996), Twenty-sixth (Int.) Symposium on Combustion, The Combustion Institute, 1997, pp. 1-26. 


\section{Figure Captions}

1. Temporal evolution of the $10 \%$ methane mass fraction isoline before, during and after the critical time. $---t=-0.02 t_{c} / t_{F},-\cdots-t=0.0 t_{c} / t_{F}$, and $\cdots \cdots \cdot \cdot t=0.02 t_{c} / t_{F}$.

2. Source and sink terms in the $\sigma$ equation (9) evaluated along the arclength in the vicinity of the critical point denoted by $10 \%$ unburnt methane mass fraction before, during and after the critical time: - production, ........ normal restoration, ----- reactive restoration, and --- dissipation, a) $t=-0.02 t_{c} / t_{F}$, b) $t=0.0 t_{c} / t_{F}$, and c) $t=$ $0.36 t_{c} / t_{F}$.

3. Source and sink terms in the $\sigma$ equation (9) evaluated along the flame normal $\left(\mathrm{n}_{1}\right.$ shown in Fig. 1) at the critical point. - production, ….... normal restoration, ---- reactive restoration, and --- dissipation. 


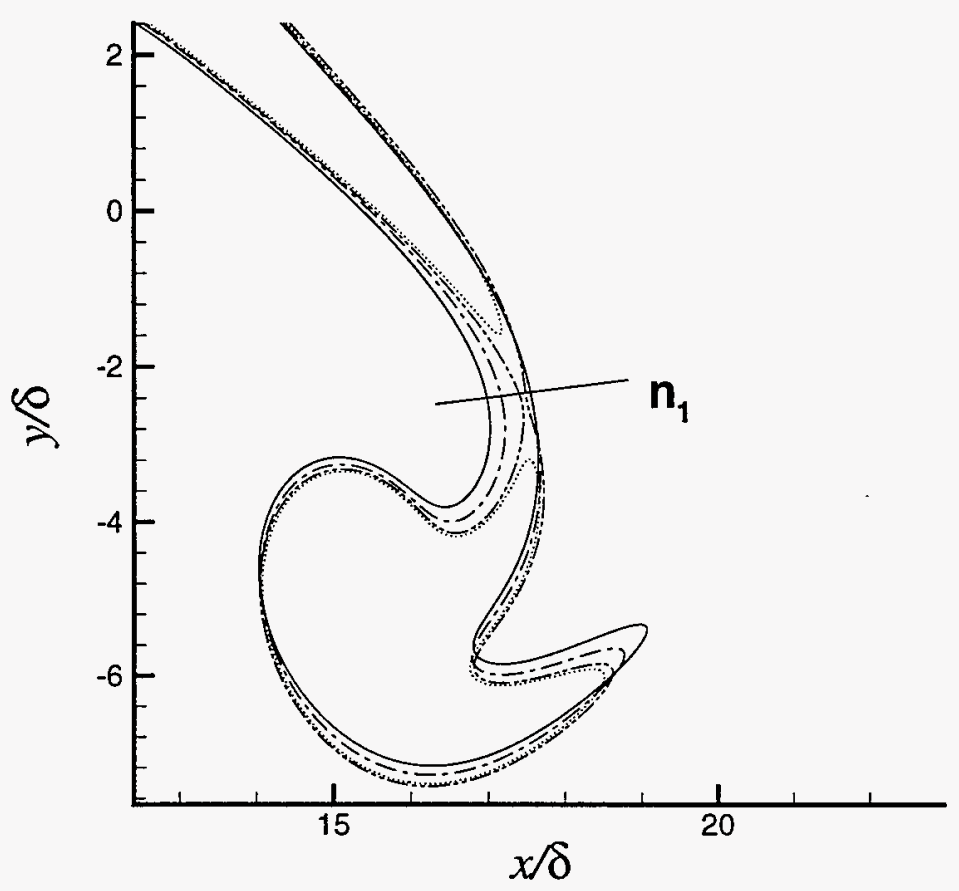

Figure 1: Temporal evolution of the $10 \%$ methane mass fraction isoline before, during and after the critical time. $---t=-0.02 t_{c} / t_{F},-.-t=0.0 t_{c} / t_{F}$, and $\cdots \cdots \cdots \cdot t=0.02 t_{c} / t_{F}$. 

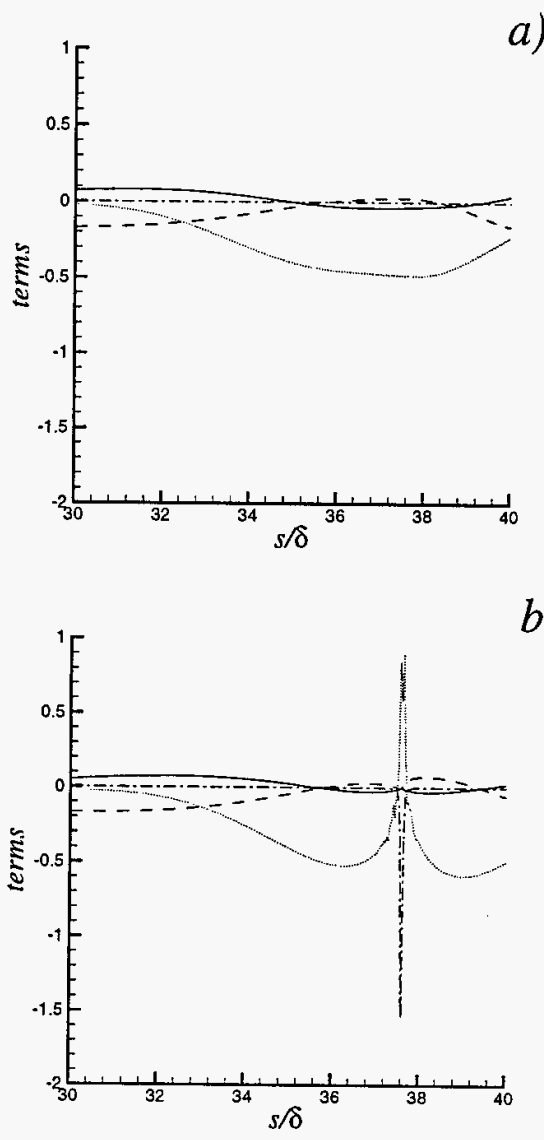

b)

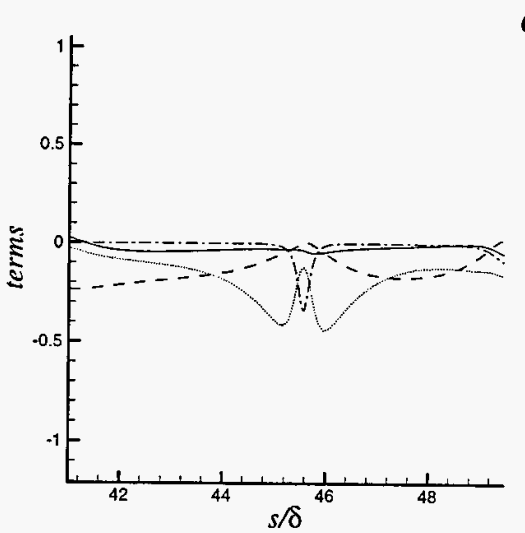

c)

Figure 2: Source and sink terms in the $\sigma$ equation (9) evaluated along the arclength in the vicinity of the critical point denoted by $10 \%$ unburnt methane mass fraction before, during and after the critical time: — production, ....... normal restoration, ---- reactive restoration, and --- dissipation, a) $t=-0.02 t_{c} / t_{F}$, b) $t=0.0 t_{c} / t_{F}$, and c) $t=0.36 t_{c} / t_{F}$. 


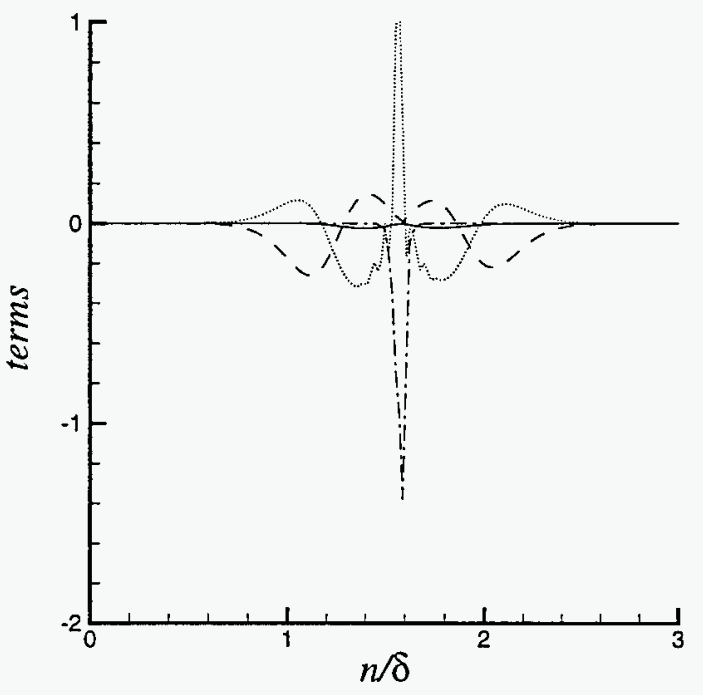

Figure 3: Source and sink terms in the $\sigma$ equation (9) evaluated along the flame normal $\left(\mathrm{n}_{1}\right.$ shown in Fig. 1) at the critical point. - production, ........ normal restoration, ---- reactive restoration, and --- dissipation. 
Report Number (14) SAND--98-8472C
CONF-980804-

Publ. Date (11) 199808 3ce

Sponsor Code (18) XF XF UC Category (19) UC-1409,DOE/ER 